\title{
Synaptic Plasticity in the Edematous Human Cerebral Cortex
}

\author{
O.J. Castejón.
}

Instituto de Investigaciones Biológicas. Facultad de Medicina. Universidad del Zulia. Maracaibo. Venezuela.

Synaptic plastic changes are fundamental events which occur spontaneuosly during development, maturity and aging processes or can be induced by injury or trauma $(1,2)$. To examine lesioninduced synaptic plasticity, cortical biopsies were taken from the frontal, parietal, temporal and occipital cortex of 18 living patients during neurosurgical treatment of brain trauma, brain tumors and vascular malformations and processed for transmission electron microscopy. Enlargement of both pre- and postsynaptic endings, irregularly shaped, lobulated, stellate and bifurcated presynaptic endings and conformational changes of dendritic spines were observed. Numerous flat, curved and invaginated axodendritic and axospinous asymmetric synapses were distinguished. A lesser proportion of axodendritic and axosomatic symmetric synapses were found. Activated or sensitized synapses showed numerous frontline spheroid synaptic vesicles, prominent dense presynaptic dense projections and increased length of synaptic membrane complex (Fig.1). Some presynaptic endings showed a stellate shape with large pedicules containing synaptic vesicles and microtubules. Other presynaptic endings appeared bifurcated giving off digitiform processes which established asymmetric septate synaptic connections. This latter observation supports the existence of dividing synapses in the oedematous cerebral cortex. Same presynaptic endings showed numerous synaptic vesicles open to the synaptic cleft, suggesting very activated synapses. Varicose and oedematous dendritic shafts with sessile, mushroom, long neck and bifurcating spines were distinguished mainly in the neuropil of parietal and frontal cortex of patients under study. We observed asymmetric synaptic contacts with round and neckless spines which show an extensive and prominent actin filamentous network anchored to the postsynaptic membrane. Asymmetric synaptic contacts with prominent synaptic densities were also seen on small, long and robust bifurcating spines. A dilated spine apparatus was consistently found in most spines. Perforated synapses (Fig.2), multiple synapses and serial synapses were also found evincing synaptic splitting and formation of new synaptic connections. The overall images suggest increased number of excitatory circuits, which were correlated with the tonico-clonic convulsion or post traumatic seizures observed in some patients. Numerous coated vesicles were observed in pre- and postsynaptic structures. Increased number of polyribosomes were found in the dendritic shafts. The dilated spine apparatus, the coated vesicles and the increased number of polyribosomes seem to represent a system for synthesis, transport and storage of synaptic proteins for the formation of new synapses. Coexisting synaptic plasticity and synaptic degeneration were observed in the patients under study. Dendritic and astrocyte synapse-like junctions were also characterized.

\section{References}

[1] O.J. CASTEJON et al. J. Neurosurg. Sci., 39, (1995) 47-65.

[2] T.A. JONES. J. Comp. Neurol., 414, (1999) 57-66.

[3] This research was supported by CONDES-LUZ. 

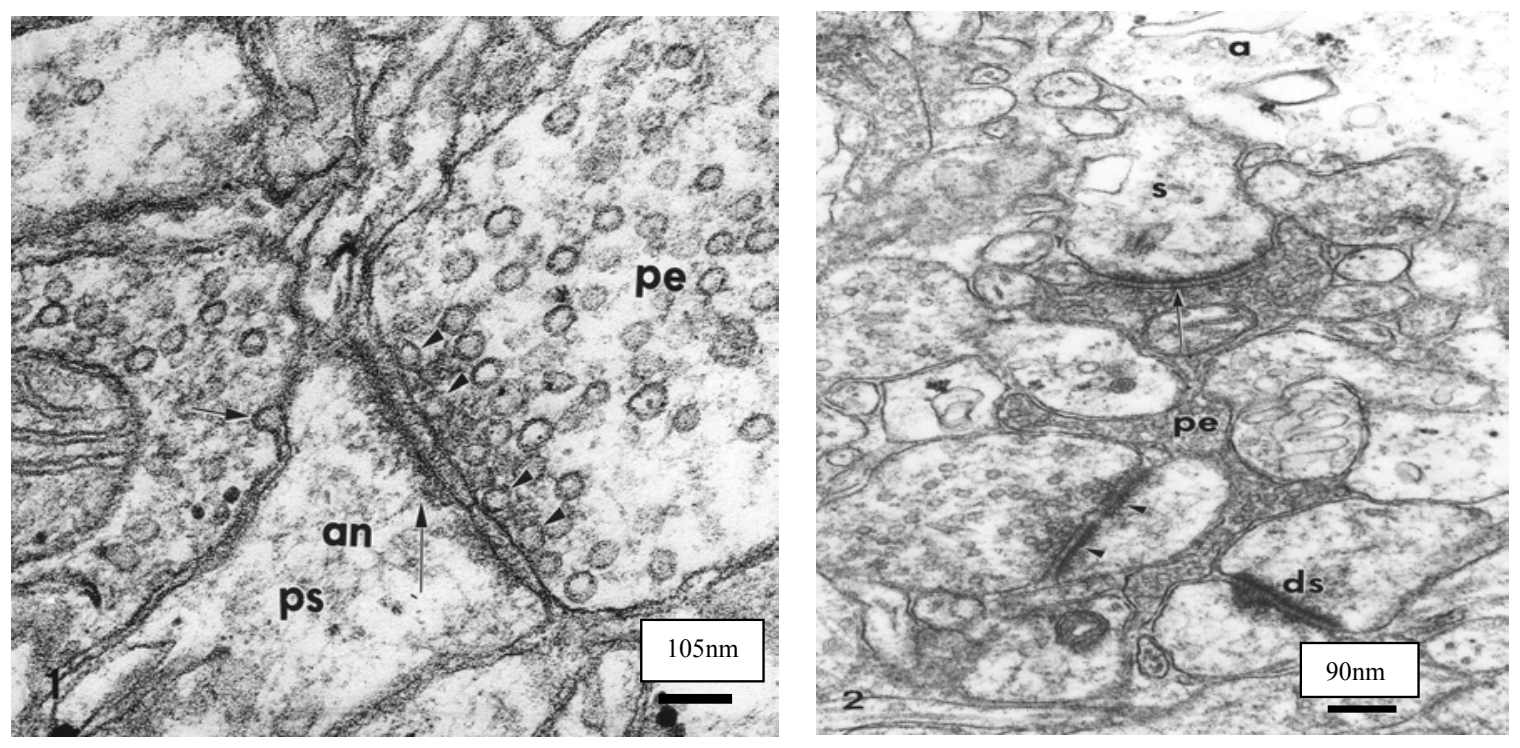

Fig. 1. Case No. 1. Right parietal cortex. Cerebellar syndrome. Axospinous dendritic asymmetric synapse showing numerous front-line and open synaptic vesicles in the presynaptic ending (pe) aggregated toward the presynaptic membrane (arrowheads). An actin- filament network (an) anchored to the postsynaptic density (long arrow) is observed in the postsynaptic spine (ps). A septate-like structure appears in the synaptic cleft joining the pre- and postsynaptic membranes. A neighbouring presynaptic ending shows an endocytic vesicle formation (short arrow). X 105.000.

Fig. 2. Case No. 7. Right parietal cortex. Anomaly of anterior cerebral artery. A lobulated and dense presynaptic ending (pe) is observed establishing an asymmetric and septated synapse (arrow) with a mushroom-type dendritic spine (s). A splitted or perforated synapse (arrowheads), a degenerated synapes (ds) and a swollen astrocytic cytoplasm (a) are also distinguished. X 90.000 . 
https://doi.org/10.1017/S1431927603447168 Published online by Cambridge University Press 